\title{
Dependently Typed Programming
}

\author{
Preface
}

This special issue of Fundamenta Informaticae is devoted to the workshops Effects and Type Theory, EffTT '07 (Tallinn, Estonia, 13-14 December 2007) and Dependently Typed Programming, DTP '08 (Nottingham, UK, 18-20 February 2008), which were organized as "small workshops" of the EU FP6 IST coordination action 510996 Types for Proofs and Programs, TYPES (2004-2008).

The workshop in Tallinn had Paul B. Levy and Aleksandar Nanevski as invited speakers. In Nottingham, invited talks were delivered by Lennart Augustsson and Xavier Leroy. Contributed talks were in both instances given by both members of the TYPES consortium and interested researchers from outside the project.

The issue features three articles on the topics of the workshops. All three demonstrate that the emerging technology of dependently typed programming is both engaging and practical.

We thank our authors and referees for their diligent work. Our colleagues in Tallinn and Nottingham deserve thanks for their help in organizing the two events.

Thorsten Altenkirch

School of Computer Science, University of Nottingham, Jubilee Campus, Wollaton Road, Nottingham NG8 1BB, U.K.

txa@cs.nott.ac.uk

Tarmo Uustalu

Institute of Cybernetics, Tallinn University of Technology,

Akadeemia tee 21, EE-12618 Tallinn, Estonia

tarmo@cs.ioc.ee

(Editors of the issue)

Nottingham-Tallinn, April 2010 